\title{
Spondyloepiphyseal dysplasia tarda, Kohn type
}

INSERM

\section{Source}

INSERM. (1999). Orphanet: an online rare disease and orphan drug data base.

Spondyloepiphyseal dysplasia tarda, Kohn type. ORPHA:163665

Spondyloepiphyseal dysplasia tarda, Kohn type is characterized by short trunk dwarfism, progressive involvement of the spine and epiphyses and mild-to-moderate intellectual deficit. 